\title{
MAGNETIC BRAKING OF LATE-TYPE STARS
}

\author{
L. MESTEL \\ Astronomy Centre, University of Sussex, \\ Falmer, Brighton, England BN1 9QH
}

\begin{abstract}
This report deals with the rotational history of late-type stars subsequent to their reaching the main sequence. The question of relevance to this Symposium is whether detailed comparison of magnetic braking theory with observations of young stellar clusters will yield any constraints on dynamo activity, in particular on the relation between the surface field strength $B_{\mathrm{s}}$ and the angular velocity $\Omega$.
\end{abstract}

\section{Magnetic braking by winds}

If not subject to a torque, outflowing gas remembers the angular momentum with which it left the stellar surface, and so its rotation lags behind the angular velocity of the star. The initially poloidal magnetic field carried out by the gas is therefore twisted, and the poloidal-plus-toroidal field exerts a magnetic torque on the gas. In a steady state, there is a constant outflow of angular momentum, carried jointly by the gas and by the moment of the Maxwell stresses. The condition that the solution be non- singular fixes the rate of outflow to be equivalent to effective corotation out to the Alfvénic surface $S_{\mathrm{A}}$ : the stellar angular momentum $J$ declines at the rate

$$
-\frac{d J}{d t} \simeq \frac{8 \pi}{3}\left(\rho v r^{2}\right)_{\mathrm{A}} \Omega r_{\mathrm{A}}^{2}=\frac{2}{3}\left(B r^{2}\right)_{\mathrm{A}}^{2} \frac{\Omega}{v_{\mathrm{A}}}
$$

where on $S_{\mathrm{A}}$ the wind speed $v$, the density $\rho$ and the poloidal field $B$ are related by

$$
4 \pi \rho_{\mathrm{A}} v_{\mathrm{A}}^{2}=B_{\mathrm{A}}^{2} .
$$

In the simplest model (Weber \& Davis 1967), the poloidal field is assumed radial and the wind is thermally driven (centrifugal effects are assumed ignorable); equation (1) then reduces to

$$
-\frac{d J}{d t} \simeq \frac{2}{3}\left(B_{\mathrm{s}} r_{\mathrm{s}}^{2}\right)^{2} \frac{\Omega}{\lambda a}
$$

where $a$ is the sound speed of the nearly isothermal corona and $\lambda$ is a slowly-varying constant $\simeq 2-3$.

If now the linear "dynamo" relation $B_{\mathrm{s}} \propto \Omega$ is postulated, $a$ is assumed independent of $\Omega$ and if also the coupling between radiative core and convective envelope is strong enough for the star to rotate nearly uniformly, then (3) becomes

$$
-\frac{d \Omega}{d t}=\kappa \Omega^{3}
$$

with $\kappa$ a function just of the spectral type. Equation (4) integrates to

$$
\Omega=\left(\Omega_{0}^{-2}+2 \kappa t\right)^{-1 / 2}
$$


with $\Omega_{0}$ the value on the zero-age main sequence. Asymptotically,

$$
\Omega=\left(\frac{1}{2 \kappa}\right)^{1 / 2} \frac{1}{t^{1 / 2}}
$$

- "Skumanich's law", inferred originally from observations of rotations of late-type stars in the Pleiades cluster $\left(7 \times 10^{7} \mathrm{y}\right)$ and in the Hyades cluster $\left(6 \times 10^{8} \mathrm{y}\right)$, and from the rotation of the $\operatorname{Sun}\left(4.5 \times 10^{9} \mathrm{y}\right)$.

Even for slow rotators, the equation (1) needs to be modified to take account of the non-radial field structure well within $S_{\mathrm{A}}$, with the X-ray bright "dead zone" of closed field lines and so with a consequent reduction in the extent of the wind zone. In addition, for rapid rotators the nearly corotating gas within $S_{\mathrm{A}}$ feels a centrifugal acceleration which supplements or even dominates over the effect of the thermal pressure. In such a "centrifugal wind", the density $\rho_{\mathrm{s}}$ at the coronal base enters explicitly into $\dot{J}$. Both effects reduce the rate of braking somewhat; for example if the relation $B_{\mathrm{s}} \propto \Omega$ is retained and if $\rho_{\mathrm{s}} \propto B_{\mathrm{s}}$, then a rigidly rotating star spins down according to $\dot{\Omega} \propto-\Omega^{p}$ with $2<p<3$ (Mestel \& Spruit 1987). If the dynamo "saturates" at $\tilde{\Omega}$, then for $\Omega>\tilde{\Omega}$ a much weaker dependence on $\Omega$ results with $p$ less than unity.

The growing abundance of observations of young stars will pose exacting tests on the theory of braking. The use of an asymptotic result such as (6) is acceptable for stars older than the Hyades cluster, for which the scatter in observed rotation rates is small, but in the Pleiades and the still younger cluster $\alpha$ Persei $\left(5 \times 10^{7}\right.$ y) the observed scatter shows that the effect of the initial distribution in $\Omega_{0}$ is still noticeable.

Interpretation of the observations would be complicated further if coupling between envelope and core is imperfect, as originally suggested by Stauffer \& Hartmann (1987) for the epoch between the ages of $\alpha$ Per and the Pleiades. Li Jianke (1992), following McGregor, has made a detailed study of the consequences of imperfect coupling, subject to the constraint from helioseismology that by the age of the Sun, $\Omega_{\text {core }}$ and $\Omega_{\text {envelope }}$ are close. His conclusion is that a model with "weak" coupling - defined by the condition that most of the core angular momentum is transferred to the envelope and carried away only after the age of the Hyades may just be consistent with observations, but it would require the early Sun to rotate slower by a factor 5 than the slowest rotators in the Hyades and Pleiades, as shown by photometric variations. "Intermediate" coupling - for which near solid body rotation is restored by the Hyades age - would yield too large a mean rotation and too big a dispersion in the Hyades. He concludes that the most plausible picture retains strong coupling (near solid body rotation) throughout all the lifetime after the zero-age main sequence, but tries to fit to the observations through a combination of a scatter in $\Omega_{0}$ and a saturation of dynamo action while $\Omega>\Omega_{0}$.

\section{Dynamo saturation}

The first attempt on these lines (Cameron \& $\mathrm{Li} 1992$, in preparation) adopts the Weber-Davis formula (3) (no centrifugal driving, no dead zones):

$$
\dot{\Omega}=-\kappa \Omega^{3} \text { for } \Omega<\tilde{\Omega}, t>\tilde{t}
$$




$$
\dot{\Omega}=-\kappa \tilde{\Omega}^{2} \Omega \text { for } \Omega>\tilde{\Omega}, 0<t<\tilde{t}
$$

with $\kappa(B-V)$ again. If $\Omega_{0}>\tilde{\Omega}$, then

$$
\begin{aligned}
& \Omega=\Omega_{0} e^{-\kappa \tilde{\Omega}^{2} t}, 0<t<\tilde{t} \\
& \Omega=\left[\tilde{\Omega}^{-2}+2 \kappa(t-\tilde{t})\right]^{-1 / 2}, T>\tilde{t}
\end{aligned}
$$

and

$$
\tilde{t}=\left(\frac{1}{\kappa \tilde{\Omega}^{2}}\right) \ln \left(\frac{\Omega_{0}}{\tilde{\Omega}}\right)
$$

while if $\Omega_{0}<\tilde{\Omega}$

$$
\Omega=\left[\Omega_{0}^{-2}+2 \kappa t\right]^{-1 / 2}, t>0 .
$$

The braking constant $\kappa$ for a star of given $(B-V)$ is estimated from observations in two ways. As noted, the weak dispersion in the Hyades implies that the effect of $\Omega_{0}$ has vanished implying, by $(6), \kappa=1 / 2 \bar{\Omega}_{\mathrm{H}}^{2} t$. A more sensitive estimate applies equation (12) to the fastest and slowest rotators in the Pleiades and Hyades. The two methods yield similar values for $\kappa$. At $5 \times 10^{9}$ y the predicted rotation is close to the solar value; also, the value for $\kappa$ is consistent with the theoretical value from (3) when observed solar estimates for $B_{\mathrm{s}}$ and $a$ are used.

Curves for $\Omega(t)$ for a solar-type star are shown in the Figure. The lower curve corresponds to an $\Omega_{0}$ that makes the curve pass through the slowest rotators observed in the Pleiades. The upper track begins with $\Omega_{0}$ at the centrifugal break-up limit for the colour studied. The saturation value $\tilde{\Omega}$ can be adjusted to yield a tolerable fit to the fastest rotators in both $\alpha$ Per and the Pleiades.

For solar-type stars, the estimated $\tilde{\Omega} \simeq 18 \Omega_{\odot}$. This is $3-4$ times the value given by Vilhu (1984) from the observed saturation of chromospheric UV and optical emission line fluxes and of coronal X-rays. Similarly, Saar (1991) finds that the filling factor of surface magnetic fields in lower main sequence stars saturates near Vilhu's chromospheric indicators. This is to be expected, as Saar's unpolarized Zeeman technique is most sensitive to the bright photospheric network, which is closely associated with plage activity. However, the amplitude of photometric variability is observed in both $\alpha$ Persei and the Pleiades to be strongly correlated with angular velocity, implying a bigger coverage of cool spots, and so of $\mathrm{kG}$ fields, which do not show up in the Zeeman technique: for example, in two stars with similar chromospheric activity but with respectively 7 - day and $1 / 2$-day rotation periods, the spot coverage is far greater in the rapid rotator. Thus the value of $\tilde{\Omega}$ higher than the Vilhu-Saar estimate is prima facie to be expected.

However, the treatment has ignored the effects of centrifugal driving at high rotations, which as noted reduces the efficiency of braking and so acts to force up the value of $\tilde{\Omega}$. Preliminary results suggest that the reasonably good fit found for the upper curve may then disappear, unless the cluster ages are doubled. It is perhaps significant that the models constructed for early-type stars by Maeder \& Meynet (1989) - with significant convective overshoot - lead to doubled ages for the clusters. 


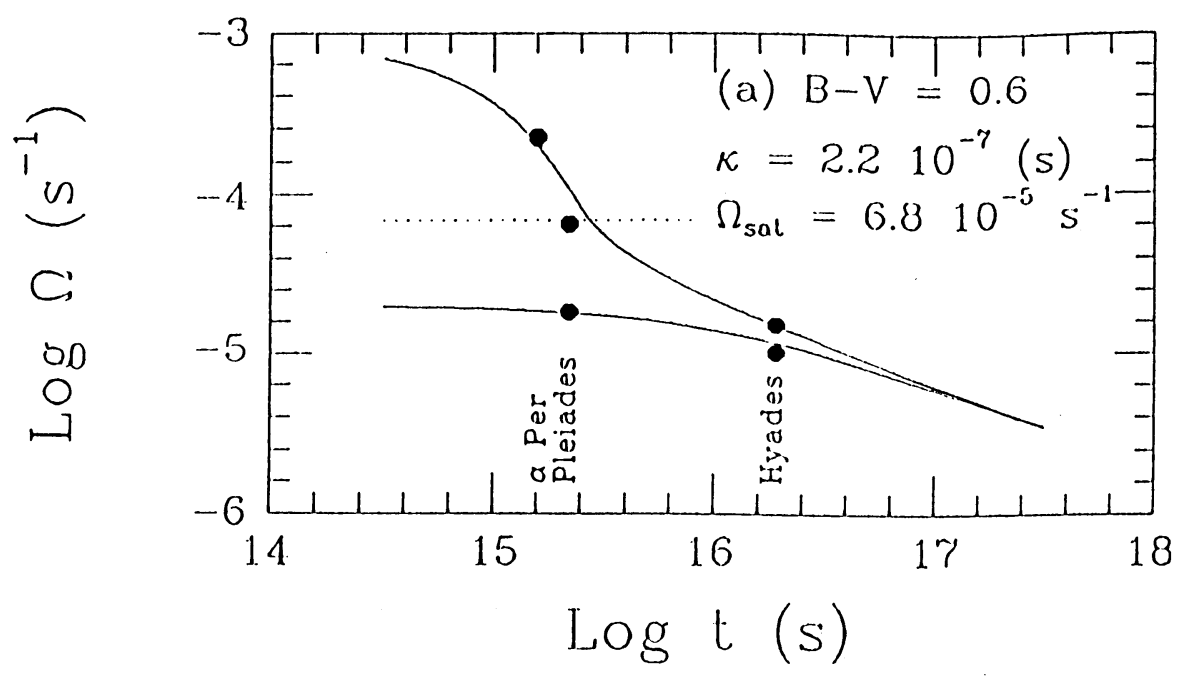

Figurc 1: Rotational cvolution tracks for rapilly and slowly rotating solar-type stars, using a Webcr-Davis model with dynamo saturation, from Camcron \& $\mathrm{Li}$ (1092).

\section{Conclusions}

1. 'To get a reliable estimate for the saturation value $\tilde{\Omega}$, we need a carcful comparison of braking theory with obscrvation.

2. The cvidence is for a distribution of $\Omega_{0}$ values on the zcro-age main scquence, so focussing at,tention on the latter stages of star formation and in particular on pre-main scquence rotational cvolution.

3. A tentative conclusion is that there is no compclling reason for corc-cnvelope decoupling in latc-type stars, or for an "cxotic", morc cfficient braking proccss.

\section{References}

Li, J., 1092, PhD thesis, University of Sussex, Brighton, England Macder A., Mcynet G., 1989, A\&A, 210, 155

Mestel L., Spruit H. C., 1987, MNRAS, 226, 57

Saar S. H., 1991, in Tuominen I., Moss D., Rüdiger G., cds, The Sun and Cool

Stars: Activity, Magnetism, Dynamos. Springer, Bcrlin, p. 389

Stauffer J. R., Hartmann L. W., 1987, ApJ, 318, 337

Vilhu O., 1984, A\&A, 133, 117

Weber E. J., Davis L., 1967, ApJ, 148, 217 